\title{
Equivariant $K$-Theory and Maps between Representation Spheres
}

\author{
Dedicated to Professor Yasutoshi Nomura on his 60th birthday
}

By

\section{Katsuhiro KomIYA*}

\section{§1. Introduction and Statement of Results}

The equivariant $K$-theory has been successfully employed in the study of equivariant maps by Marzantowicz [5], Liulevicious [7] and Bartsch [3]. In the present paper, using the equivariant $K$-theory, we will obtain a necessary condition for the existence of $G$-maps $S U \rightarrow S W$, where $S U$ and $S W$ are the unit spheres of unitary representations $U$ and $W$, respectively, of a compact Lie group $G$.

From Atiyah [1], [2] or Segal [8] we can see that the equivariant $K$-ring $K_{G}(S U)$ of $S U$ is isomorphic to $R(G) /\left(\lambda_{-1} U\right)$, the complex representation ring $R(G)$ divided by the ideal $\left(\lambda_{-1} U\right)$ generated by the Euler class $\lambda_{-1} U$ of $U$ in $K_{G}(\mathrm{pt})=R(G)$. If there exists a $G$-map $\eta: S U \rightarrow S W$, then we obtain a ring homomorphism $\eta: R(G) /\left(\lambda_{-1} W\right) \rightarrow R(G) /\left(\lambda_{-1} U\right)$ which coincides with the homomorphism induced from the identity on $R(G)$. This implies that the condition $\lambda_{-1} W \in\left(\lambda_{-1} U\right)$ is necessary for the existence of $G$-maps $S U \rightarrow S W$. If $G$ is abelian, we will reduce this condition to more exlicit form.

Let $S^{1}=\{z \in C|| z \mid=1\}$ be the circle group of complex numbers with absolute value 1 , and $Z_{n}$ the cyclic group of order $n$ considered as a subgroup of $S^{1}$. For any integer $i$ let $S^{1}$ and $Z_{n}$ act on $V_{1}=C$ via $(z, v) \mapsto z^{\prime} v$ for $z \in S^{1}\left(\right.$ or $\left.Z_{n}\right)$ and $v \in V_{l}$. A compact abelian group $G$ decomposes into a cartesian product

$$
G=T^{h} \times Z_{n_{l}} \times \cdots \times Z_{n_{l}},
$$

where $T^{k}=S^{1} \times \cdots \times S^{1}$, the cartesian product of $k$ copies of $S^{1}$. Letting $\gamma$ be a sequence $\left(a_{1}, \ldots, a_{h}, b_{l}, \ldots, b_{l}\right)$ of integers, denote by $V_{\gamma}$ the tensor product

$$
V_{a_{1}} \otimes \cdots \otimes V_{a_{k}} \otimes V_{b_{1}} \otimes \cdots \otimes V_{b_{1}},
$$

Communicated by K. Saito, December 19, 1994.

1991 Mathematics Subject Classifications: 55N15, 57S99

* Department of Mathematics, Yamaguchi University, Yamaguchi 753, Japan 
which can be considered as a representation of $G$ in a natural way. Let $\Gamma$ be the set of sequences

$$
\gamma=\left(a_{1}, \ldots, a_{k}, b_{1}, \ldots, b_{l}\right)
$$

with $a_{1}, \ldots, a_{k} \in \mathbb{Z}$ and $0 \leq b_{j} \leq n_{\jmath}-1$ for $1 \leq j \leq l$. The set $\left\{V_{\gamma} \mid \gamma \in \Gamma\right\}$ gives a complete set of irreducible unitary representations of $G$, and so any unitary representation $U$ of $G$ decomposes into a direct sum

$$
U=\underset{\gamma \in \Gamma}{\bigoplus} V_{\gamma}^{u(\gamma)},
$$

where $u(\gamma)$ is a nonnegative integer and $V_{\gamma}^{u(\gamma)}$ denotes the direct sum of $u(\gamma)$ copies of $V_{\gamma}$. We can easily see that the fixed point set $U^{G}$ of $U$ is $\{0\}$ if and only if $u(\gamma)=0$ for $\gamma=(0, \ldots, 0)$. Let

$$
|\gamma|=\left|a_{1}\right|+\cdots+\left|a_{h}\right|+b_{1}+\cdots+b_{l}
$$

for any $\gamma=\left(a_{1}, \ldots, a_{h}, b_{1}, \ldots, b_{l}\right) \in \Gamma$.

We are now in a position to state our main theorem.

Theorem $\mathbb{1} . \mathbb{1}$. Let $U$ and $W$ be unitary representations of a compact abelian group $G$, and decompose into

$$
U=\underset{\gamma \in \Gamma}{\bigoplus} V_{\gamma}^{u(\gamma)} \text { and } W=\underset{\gamma \in \Gamma}{\bigoplus} V_{\gamma}^{n(\gamma)} .
$$

Assume that there exists a G-map $S U \rightarrow S W$. Then

(1) if $\operatorname{dim} U=\operatorname{dim} W$, then there is an integer $m$ such that

$$
\prod_{\gamma \in \Gamma}|\gamma|^{n(\gamma)} \equiv m \prod_{\gamma \in \Gamma}|\gamma|^{u(\gamma)} \bmod d,
$$

where $d$ is the greatest common divisor of $n_{1}, \ldots, n_{l}$, (if $l=0$, then assume $\left.d=0\right)$,

(2) if $\operatorname{dim} U>\operatorname{dim} W$, then

$$
\prod_{\gamma \in \Gamma}|\gamma|^{n(\gamma)} \equiv 0 \bmod d .
$$

From this theorem we obtain the following two corollaries.

Corollary 1.2 (cf. Liulevicious [7], Bartsch [4], Marzantowicz [6]). Let $U$ and $W$ be representations of $G=T^{k}$ with $W^{G}=\{0\}$. If there exists a $G$-map $S U \rightarrow S W$, then $\operatorname{dim} U \leq \operatorname{dim} W$.

Corollary 1.3 (Liulevicious [7], Marzantowicz [6]). Let $U$ and $W$ be representations of $G=\mathbb{Z}_{n}$ with $n$ any. If $G$ acts freely on $S W$ and if there exists a $G$ map $S U \rightarrow S W$, then $\operatorname{dim} U \leq \operatorname{dim} W$. 
Remark 1.4. If $U$ is an orthogonal representation of $G=T^{k}$ or $Z_{n}$ with $n$ odd and if $U^{G}=\{0\}$, then $U$ can be considered a unitary representation. In general, if $U$ is orthogonal then $U \oplus U$ becomes unitary. Since the join of two $G$ maps $S U \rightarrow S W$ gives a $G$-map

$$
S(U \oplus U)=S U * S U \rightarrow S W * S W=S(W \oplus W),
$$

Corollaries 1.2 and 1.3 follow for orthogonal representations $U$ and $W$.

Remark 1.5. We should refer to a recent paper [6] of Marzantowicz. Using the Borel cohomology theory, he also studies equivariant maps between representation spheres, and obtains a necessary condition for the existence of such maps. A detailed study is done for the case of $G=T^{k}$ or $Z_{p}^{k}\left(=Z_{p} \times \cdots \times \mathbb{Z}_{p}\right)$. It is also shown that his condition is sufficient in some case.

\section{§2. A Necessary Condition in Terms of the Euler Classes}

Let $U$ be a unitary representation of a compact Lie group $G$. The sequence

$$
\cdots \rightarrow K_{G}^{n}(D U, S U) \rightarrow K_{G}^{n}(D U) \rightarrow K_{G}^{n}(S U) \rightarrow K_{G}^{n+1}(D U, S U) \rightarrow \cdots
$$

is the long exact sequence of the equivariant $K$-theory $K_{G}^{\prime}$ for the pair $(D U, S U)$ of the unit disk $D U$ and the unit sphere $S U$ of $U$. Segal [8; Proposition 3.2] or Atiyah [2] gives the Thom isomorphism

$$
\varphi: K_{G}(\mathrm{pt}) \rightarrow K_{G}(U)=K_{G}(D U, S U)
$$

such that $\varphi \varphi(\xi)=\xi \cdot \lambda_{-1} U$ for $\xi \in K_{G}(\mathrm{pt})$, where $\varphi: K_{G}(U) \rightarrow K_{G}(\mathrm{pt})$ is the homomorphism induced from the inclusion map $\varphi:\{\mathrm{pt}\} \rightarrow U$,

$$
\lambda_{-1} U=\sum_{1}(-1)^{\prime} \Lambda^{\prime} U \in K_{G}^{0}(\mathrm{pt}),
$$

and $\Lambda^{\prime} U$ is the $i$-th exterior algebra of $U$. Since $K_{G}^{1}(D U, S U)=K_{G}^{1}(U) \cong K_{G}^{1}(\mathrm{pt})$ $=0$ and $K_{G}^{0}(\mathrm{pt}) \cong R(G)$, the sequence $(2.1)$ yields the exact sequence

$$
R(G) \rightarrow R(G) \rightarrow K_{G}(S U) \rightarrow 0,
$$

where the first homomorphism is given by multiplication by $\lambda_{-1} U$. This argument is done in the same manner as in Atiyah [1; Lemma 2.7.4, Corollary 2.7.5] where $G$ is finite abelian.

From the exact sequence (2.2) we obtain

Proposition 2.3. $K_{G}(S U) \cong R(G) /\left(\lambda_{-1} U\right)$.

Let $\eta: S U \rightarrow S W$ be a G-map for representations $U$ and $W$ of $G$. Since the sequence (2.1) is functorial, we see that the composite 


$$
R(G) /\left(\lambda_{-1} W\right) \cong K_{G}(S W) \stackrel{\eta}{\longrightarrow} K_{G}(S U) \cong R(G) /\left(\lambda_{-1} U\right)
$$

coincides with the homomorphism induced from the identity on $R(G)$. This implies the following.

Proposition 2.4. If there exists a $G$-map $S U \rightarrow S W$, then $\lambda_{-1} W \in\left(\lambda_{-1} U\right)$ in $R(G)$.

\section{§3. Calculation of $K_{G}(S U)$}

In this section we will calculate the ring $K_{G}(S U)$ for the case where $G$ is abelian.

We first recall the following facts about the complex representation rings of $G$

(1) $R\left(S^{1}\right) \cong \mathbb{Z}\left[x, x^{-1}\right] /\left(1-x x^{-1}\right)$, in which the representation $V_{1}$ corresponds to $x^{\prime}$ if $i \geq 0$ and to $\left(x^{-1}\right)^{-1}$ if $i \leq 0$.

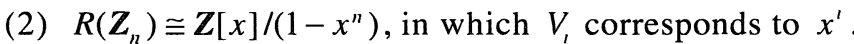

(3) $R\left(G_{1} \times G_{2}\right) \cong R\left(G_{1}\right) \otimes R\left(G_{2}\right)$.

From these facts we obtain

Proposition 3.1. If $G=T^{n} \times \mathbb{Z}_{n_{1}} \times \cdots \times \mathbb{Z}_{n_{1}}$ is a compact abelian group, then $R(G) \cong \mathbb{Z}\left[x_{1}, x_{1}^{-1}, \ldots, x_{h}, x_{h}^{-1}, y_{1}, \ldots, y_{l}\right] /(X, Y)$,

where

$$
\begin{aligned}
& X=\left\{1-x_{\imath} x_{t}^{-1} \mid 1 \leq i \leq k\right\}, \\
& Y=\left\{1-y_{J}^{n_{1}} \mid 1 \leq j \leq l\right\},
\end{aligned}
$$

and $(X, Y)$ is the ideal generated by $X \cup Y$. The isomorphism sends the representation $V_{\gamma}$ to the monomial $x_{1}^{a_{1}} \cdots x_{k}^{a_{k}} y_{1}^{b_{1}} \cdots y_{l}^{b_{l}}$ if $\gamma=\left(a_{1}, \ldots, a_{h}, b_{1}, \ldots, b_{l}\right)$.

Since $\lambda_{-1}$ is multiplicative, i.e., $\lambda_{-1}\left(U_{1} \oplus U_{2}\right)=\lambda_{-1} U_{1} \cdot \lambda_{-1} U_{2}$, Propositions 2.3 and 3.1 give the following.

Proposition 3.2. Let $U=\oplus_{\gamma \in \Gamma} V_{\gamma}^{u(\gamma)}$ be a unitary representation of $G=$ $T^{h} \times Z_{n_{1}} \times \cdots \times \mathbb{Z}_{n_{1}}$. Then

$$
K_{G}(S U) \cong Z\left[x_{1}, x_{1}^{-1}, \ldots, x_{h}, x_{h}^{-1}, y_{1}, \ldots, y_{l}\right] /\left(X, Y, z_{U}\right),
$$

where $z_{U}=\Pi_{\gamma}\left(1-(\boldsymbol{x y})^{\gamma}\right)^{u(\gamma)},(\boldsymbol{x} \boldsymbol{y})^{\gamma}=x_{1}^{a_{1}} \cdots x_{h}^{a_{h}} y_{1}^{b_{1}} \cdots y_{l}^{b_{l}} \quad$ if $\quad \gamma=\left(a_{1}, \ldots, a_{k}, b_{1}, \ldots, b_{l}\right)$, and $\left(X, Y, z_{U}\right)$ is the ideal generated by $X \cup Y \cup\left\{z_{U}\right\}$. 


\section{§4. Proof of Theorem 1.1}

In this section we will prove Theorem 1.1. Let $G=T^{k} \times Z_{n_{1}} \times \cdots \times Z_{n_{l}}$ be a compact abelian group, and

$$
U=\underset{\gamma \in \Gamma}{\bigoplus} V_{\gamma}^{u(\gamma)}, \quad W=\underset{\gamma \in \Gamma}{\oplus} V_{\gamma}^{u(\gamma)}
$$

its unitary representations. Assume that there exists a $G$-map $\eta: S U \rightarrow S W$. If $W^{G} \neq\{0\}$, then the theorem is trivially valid. So we assume $W^{G}=\{0\}$.

For the representation

$$
V_{\gamma}=V_{a_{1}} \otimes \cdots \otimes V_{a_{k}} \otimes V_{b_{1}} \otimes \cdots \otimes V_{b_{1}},
$$

let

$$
\bar{V}_{\gamma}=V_{\left|a_{1}\right|} \otimes \cdots \otimes V_{\left|a_{k}\right|} \otimes V_{b_{1}} \otimes \cdots \otimes V_{b_{1}}
$$

and

$$
\bar{U}=\underset{\gamma \in \Gamma}{\oplus} \bar{V}_{\gamma}^{u(\gamma)}, \quad \bar{W}=\underset{\gamma \in \Gamma}{\oplus} \bar{V}_{\gamma}^{u(\gamma)} .
$$

Since $V_{a} \cong V_{|a|}$ as real representations, we see $U \cong \bar{U}$ and $W \cong \bar{W}$. Therefore $\eta: S U \rightarrow S W$ induces a $G$-map $\bar{\eta}: S \bar{U} \rightarrow S \bar{W}$, and then $\bar{\eta}$ induces a ring homomorphism $\bar{\eta}: K_{G}(S \bar{W}) \rightarrow K_{G}(S \bar{U})$. From Proposition 3.2 we obtain a ring homomorphism

$\bar{\eta}^{\prime}: Z\left[x_{1}, x_{1}^{-1}, \ldots, x_{h}, x_{h}^{-1}, y_{1}, \ldots, y_{l}\right] /\left(X, Y, \bar{z}_{W}\right) \rightarrow Z\left[x_{1}, x_{1}^{-1}, \ldots, x_{h}, x_{k}^{-1}, y_{1}, \ldots, y_{l}\right] /\left(X, Y, \bar{z}_{U}\right)$,

where $X$ and $Y$ are as given in Proposition 3.1,

$$
\bar{z}_{U}=\prod_{\gamma \in \Gamma}\left(1-\overline{\boldsymbol{x y}}^{\gamma}\right)^{u(\gamma)}, \quad \bar{z}_{W}=\prod_{\gamma \in \Gamma}\left(1-\overline{\boldsymbol{x y}}^{\gamma}\right)^{w(\gamma)},
$$

and

$$
\overline{\boldsymbol{x y}}^{\gamma}=x_{1}^{\left|a_{1}\right|} \cdots x_{h}^{\left|a_{k}\right|} y_{1}^{b_{1}} \cdots y_{1}^{b_{1}} .
$$

As in Proposition 2.4, we see $\bar{z}_{W} \in\left(X, Y, \bar{z}_{U}\right)$. Then there are polynomials $f_{l}(1 \leq j \leq l+1)$ in $\mathbb{Z}\left[x_{1}, x_{1}^{-1}, \ldots, x_{h}, x_{h}^{-1}, y_{1}, \ldots, y_{l}\right]$ such that

$$
\bar{z}_{W}=\sum_{j=1}^{l} f_{l} \cdot\left(1-y_{l}^{n^{\prime}}\right)+f_{l+1} \cdot \bar{z}_{U}
$$

in $Z\left[x_{1}, x_{1}^{-1}, \ldots, x_{k}, x_{h}^{-1}, y_{1}, \ldots, y_{l}\right] /(X)$. Multiplying (4.1) by $x_{1}^{m_{1}} \cdots x_{k}^{m_{k}}$ for sufficiently large $m_{1}, \cdots, m_{h}>0$, we obtain

$$
x_{1}^{m_{1}} \cdots x_{h}^{m_{k}} \bar{z}_{W}=\sum_{j=1}^{l} \tilde{f}_{l} \cdot\left(1-y_{l}^{n_{l}}\right)+\tilde{f}_{l+1} \cdot \bar{z}_{U}
$$


in $\mathbb{Z}\left[x_{1}, \ldots, x_{h}, y_{1}, \ldots, y_{l}\right]$, where $\tilde{f}_{,}(1 \leq j \leq l+1)$ are polynomials in $\mathbb{Z}\left[x_{1}, \ldots, x_{h}, y_{1}\right.$, $\left.\ldots, y_{l}\right]$. Substituting $x$ for all of $x_{1}, \ldots, x_{k}, y_{1}, \ldots, y_{l}$ in (4.2), we obtain

$$
x^{m} \prod_{\gamma \in \Gamma}\left(1-x^{|\gamma|}\right)^{w(\gamma)}=\sum_{j=1}^{l} g_{J}(x)\left(1-x^{n_{\jmath}}\right)+g_{l+1}(x) \underset{\gamma \in \Gamma}{\prod}\left(1-x^{|\gamma|}\right)^{u(\gamma)},
$$

where $m=m_{1}+\cdots+m_{k}, g_{j}(x) \in \mathbb{Z}[x](1 \leq j \leq l+1)$ and $|\gamma|=\left|a_{1}\right|+\cdots+\left|a_{k}\right|+b_{1}+\cdots+b_{l}$ if $\gamma=\left(a_{1}, \ldots, a_{k}, b_{1}, \ldots, b_{l}\right)$. If $\operatorname{dim} U \geq \operatorname{dim} W$, we can divide the both sides of (4.3) by $(1-x)^{\sum u(\gamma)}$, and obtain

$$
\begin{aligned}
x^{m} & \prod_{\gamma \in \Gamma}\left(1+x+\cdots+x^{|\gamma|-1}\right)^{w(\gamma)} \\
& =h(x)+g_{l+1}(x)(1-x)^{\sum^{u(\gamma)-\sum w(\gamma)} \prod_{\gamma \in \Gamma}\left(1+x+\cdots+x^{|\gamma|-1}\right)^{u(\gamma)}},
\end{aligned}
$$

where $h(x)=\sum_{j=1}^{l} g_{j}(x)\left(1-x^{n_{1}}\right) /(1-x)^{\sum w(\gamma)} \in \mathbb{Z}[x]$. Since

$$
1-x^{n_{J}}=(1-x)\left(1+x+\cdots+x^{d_{1}-1}\right) p_{J}(x)
$$

for any divisor $d_{\text {, of }} n_{J}$ and some $p_{J}(x) \in \mathbb{Z}[x]$, we see

$$
\sum_{J=1}^{l} g_{J}(x)\left(1-x^{n_{\prime}}\right)=(1-x)\left(1+x+\cdots+x^{d-1}\right) \sum_{J=1}^{l} g_{J}(x) p_{\jmath}(x),
$$

where $d$ is the greatest common divisor of $n_{1}, \ldots, n_{l}$. Since $1-x$ and $1+x+\cdots$ $+x^{d-1}$ are prime to each other, $h(x)=\left(1+x+\cdots+x^{d-1}\right) q(x)$ for some $q(x) \in \mathbb{Z}[x]$. Therefore, substituting 1 for $x$ in (4.4), we obtain

$$
\prod_{\gamma \in \Gamma}|\gamma|^{n(\gamma)}=d \cdot q(1)+g_{l+1}(1) \prod_{\gamma \in \Gamma}|\gamma|^{u(\gamma)}
$$

if $\sum_{\gamma} u(\gamma)=\sum_{\gamma} w(\gamma)$, and

$$
\prod_{\gamma \in \Gamma}|\gamma|^{n(\gamma)}=d \cdot q(1)
$$

if $\sum_{\gamma} u(\gamma)>\sum_{\gamma} w(\gamma)$. This completes the proof of Theorem 1.1.

\section{References}

[1] Atiyah, M.F., Lectures on K-theory, Benjamin, New-York, 1967.

[2] , Bott periodicity and the index of elliptic operators, Quart. J. Math., 19 (1968), 113140.

[3] Bartsch, T., On the genus of representation spheres, Comment. Math. Helv., 65 (1990), 85-95.

[4] - On the existence of Borsuk-Ulam theorems, Topology, 31 (1992), 533-543.

[5] Marzantowicz, W., The Lefschetz number in equivariant $K$-theory, Bull. Acad. Pol. Sci., 25 (1977), 901-906.

[6] -, Borsuk-Ulam theorem for any compact Lie group, J. London Math. Soc., 49 (1994), 195-208.

[7] Liulevicious, A., Borsuk-Ulam theorems and $K$-theory degrees of maps, Springer Lecture Notes in Math., 1051 (1984), 610-619.

[8] Segal, G., Equivariant $K$-theory, Publ. Math. IHES, 34 (1968), 129-151. 